\title{
Complete Response of Para-Aortic and Lateral Pelvic Lymph Node Recurrence of Rectal Cancer Treated to S-1 Monotherapy
}

\author{
Tomonori Miyazawa, ${ }^{\mathrm{a}, \mathrm{b}}$, Norihiko Koide ${ }^{\mathrm{a}}$, Nobuhiro Fujita ${ }^{\mathrm{a}}$
}

\begin{abstract}
This report presents a case of para-aortic and lateral pelvic lymph node recurrence of rectal cancer that showed complete response to S-1 monotherapy. A 69-year-old man underwent low anterior resection for rectal cancer in 2007. Para-aortic lymph and right lateral pelvic lymph node recurrence occurred in 2008. He received a fluorouracil/folinic acid plus oxaliplatin regimen; however, G4 neutropenia and G3 fatigue were experienced. We started S-1 monotherapy as a salvage treatment. Abdominal computed tomography did not reveal any para-aortic and lateral pelvic lymph nodes recurrence after 10 cycles of S-1 monotherapy. Hence, response in this case was classified as a complete response. No recurrence was noted 36 months after the complete response. S-1 monotherapy is likely to be effective in treating patients with metastatic colorectal cancer who do not respond to standard combination chemotherapy.
\end{abstract}

Keywords: Para-aortic and lateral lymph node recurrence; Rectal cancer; S-1

\section{Introduction}

The introduction of oxaliplatin and irinotecan into combination regimens with fluorouracil (5-FU) has been a major advance in the treatment of patients with metastatic colorectal cancer (MCRC). Oxaliplatin/5-FU/folinic acid (FOLFOX-4) is the standard first-line chemotherapy in patients with MCRC $[1,2]$. The common grade $3 / 4$ adverse events associated with FOLFOX-4 are neutropenia and granulocytopenia; therefore, treatment might be discontinued because of such adverse events in patients treated with FOLFOX-4 $[1,2]$. S-1 is an oral anticancer agent containing tegafur, a

\footnotetext{
Manuscript accepted for publication January 25, 2013

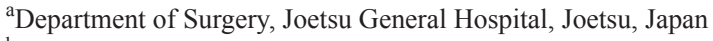

${ }^{\mathrm{b}}$ Corresponding author: Tomonori Miyazawa, 148-1 Daidofukuda, Joetsu, Niigata 943-8507, Japan. Email: tmiyazaw54@yahoo.co.jp
}

doi: http://dx.doi.org/10.4021/wjon619w metabolically activated prodrug of 5-FU, and 2 biochemical modulators [3]. Three phase 2 trials reported that S-1 monotherapy showed response similar to that of infusional 5-FU/ folinic acid as the first-line treatment in patients with MCRC [4-6]. Two studies showed that salvage S-1 monotherapy was effective and well tolerated in MCRC patients after the failure of irinotecan-based or oxaliplatin-based chemotherapy $[7,8]$. Here, we report a case of para-aortic and lateral pelvic lymph node recurrence of rectal cancer that showed complete response (CR) to S-1 monotherapy after discontinuance of FOLFOX-4 because of grade 4 adverse events.

\section{Case Report}

A 69-year-old man with mild liver dysfunction due to chronic type $\mathrm{C}$ hepatitis, underwent low anterior resection for rectal cancer in 2007. Pathological examination showed a well-differentiated adenocarcinoma invading the perirectal tissues, with metastatic involvement in 1 of the 15 lymph nodes removed (T4 N1M0/Stage IIIB), (Fig. 1). The patient underwent 5 cycles of tegafur-uracil plus oral leucovolon therapy (UFT/LV) as adjuvant chemotherapy without any adverse events. In 2008, abdominal computed tomography (CT) after UFT/LV showed swollen para-aortic and right lateral pelvic lymph nodes (Fig. 2a, b). Thus, para-aortic and pelvic lymph nodes recurrence of rectal cancer was diagnosed. We started a FOLFOX-4 regimen in March 2008. However, the patient experienced G4 neutropenia and G3 fatigue (according to the Common Terminology Criteria for Adverse Events Version 4.0), and therefore had to discontinue FOLFOX-4. We started S-1 administration $(100 \mathrm{mg} /$ day twice on days $1-14$, every 3 weeks) as salvage treatment in May 2008. Abdominopelvic CT after 5 cycles of S-1 showed reduction of the swollen para-aortic lymph nodes (Fig. 2c, d).

Another series of abdominal CT performed after 10 cycles of S-1 monotherapy showed complete disappearance of swollen para-aortic and lateral pelvic lymph nodes (Fig. 2e, f). Hence, response in this case was classified as a CR. The patient did not experience any adverse events during the S-1 monotherapy. No signs of recurrence or metastasis were noted 36 months after $\mathrm{CR}$ was confirmed. 


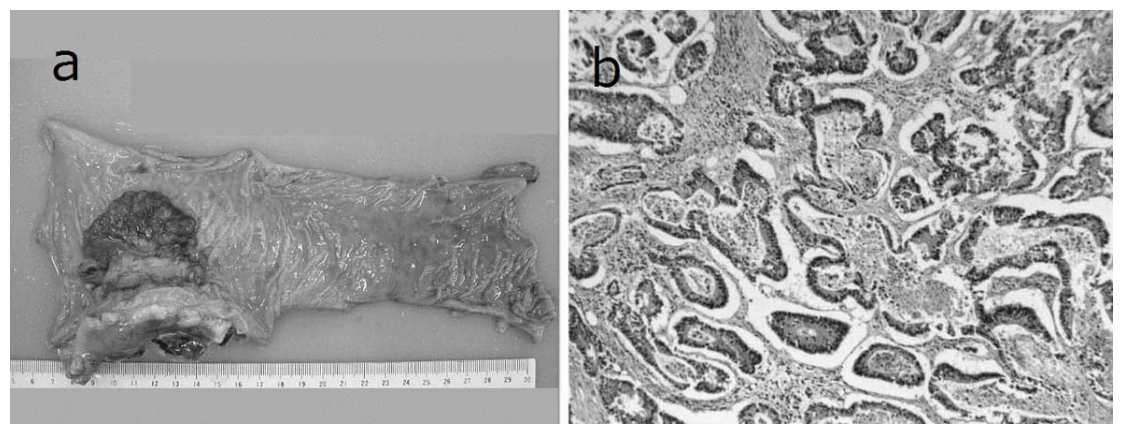

Figure 1. Resected specimen and pathological findings. a): Macroscopic appearance of surgically resected specimen showing type 2 advanced rectal cancer; b): Pathological examination showed a well-differentiated adenocarcinoma invading the perirectal tissues, with metastatic involvement in 1 of the 15 resected lymph nodes. (hematoxylin and eosin stain).
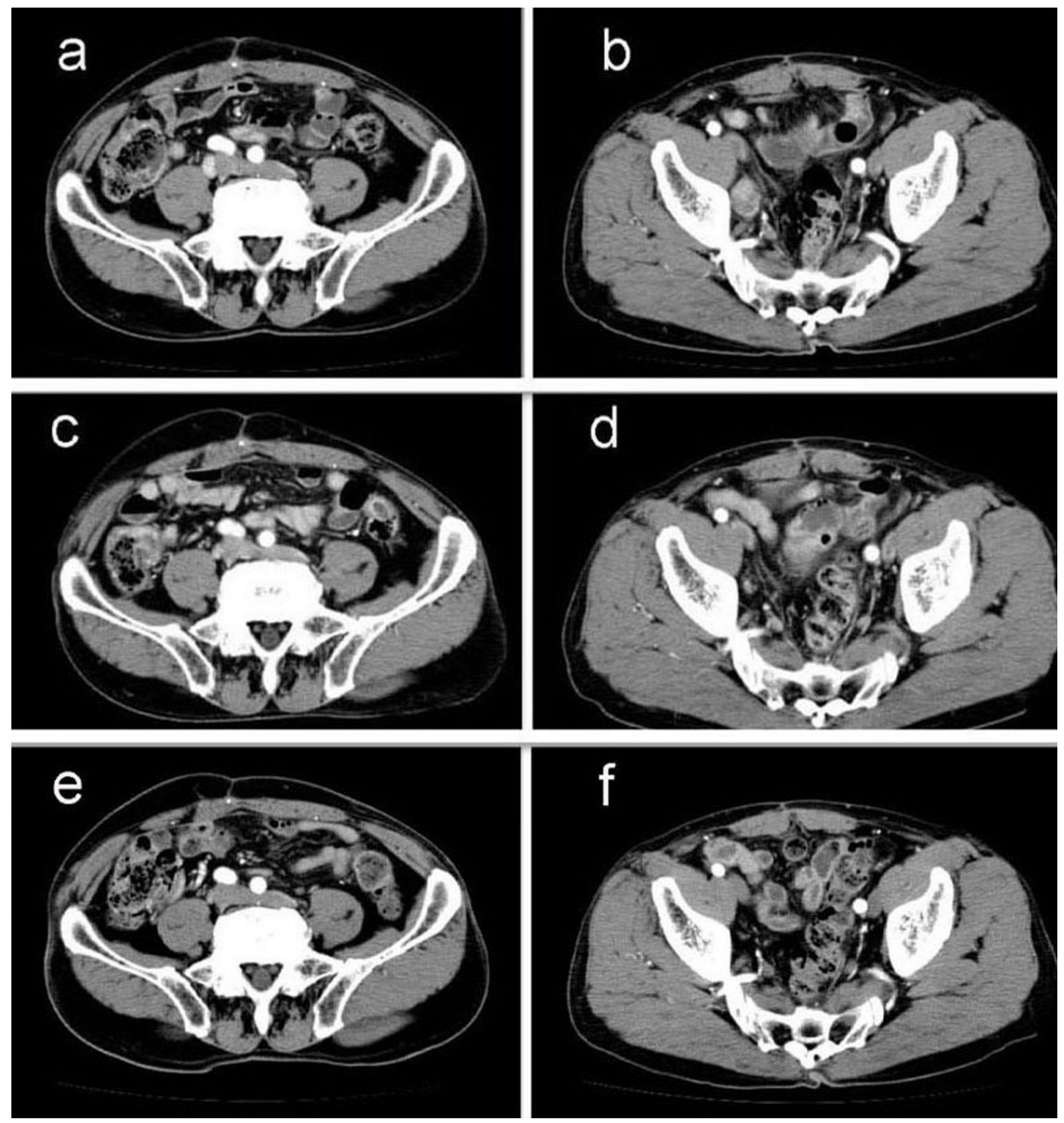

Figure 2. Abdominal computed tomography (CT) findings. a-b): CT scan before chemotherapy showing swollen paraaortic and right lateral pelvic lymph nodes; c-d): CT scan after 5 cycles of S-1 administration showing reduction in the swollen para-aortic lymph nodes; e-f): CT scan after radiation and 10 cycles of S-1 administration showing complete disappearance of swollen para-aortic and lateral pelvic lymph nodes. Hence, response in this case was classified as a complete response.

\section{Discussion}

The key drug for systemic treatment of colorectal cancer is 5-FU. The introduction of oxaliplatin and irinotecan into combination regimens with 5-FU has been a major advance in treatment of patients with MCRC. FOLFOX-4 and irinotecan/5-FU/folinic acid (FOLFIRI) regimens are the standard first-line or second-line chemotherapies in pa- 
tients with MCRC. However, these regimens involve continuous infusions of 5-FU that requires catheter replacement and regular visits to the clinic [2]. Grade $3 / 4$ neutropenia is more common with FOLFOX-4 than with 5-FU/ folinic acid $[1,2]$. Oral 5 -FU prodrugs capecitabine and UFT/LV have already been proven to show an efficacy equivalent to that of an intravenous 5-FU/folinic acid regimen in patients of MCRC, but with less toxicity than the 5-FU regimen [9-11]. Furthermore, capecitabine plus oxaliplatin therapy was noninferior to FOLFOX-4 as a first-line treatment for MCRC [2]. These oral 5-FU prodrugs have the advantage of reducing intravenous drug administration and associated visits to the clinic.

S-1 is an oral anticancer agent containing tegafur, a metabolically activated prodrug of 5-FU, with 2 biochemical modulators of 5-FU metabolism: 5-chloro-2, 4-dihydroxypyridine that inhibits 5-FU degeneration by dihydroxypyridine dehydrogenase and potassium oxonate that reduces the incidence of 5-FU-induced gastrointestinal side effects [3]. Three phase 2 trials have demonstrated that S- 1 achieved responses similar to those of infusional 5-FU/folinic acid and had acceptable toxicity profile as first-line treatment in patients with MCRC [4-6]. Shibahara et al reported equivalent efficacy and safety between S-1 and UFT/LV in patients with MCRC [12]. Two studies showed that salvage S-1 monotherapy was effective and well tolerated in MCRC patients after failure of irinotecan-based or oxaliplatin-based chemotherapy $[7,8]$. Recent studies demonstrated that the combination of S-1 and oxaliplatin or irinotecan could be an additional therapeutic options for patients with MCRC [13-15]. S-1 is usually administrated for 4 weeks, followed by a 2-week drug-free period. However, adverse reactions related to S-1 therapy commonly begin to appear in 2 - 3 weeks after treatment starts. Therefore, a 2 -week regimen of S-1 followed by a 1-week drug- free period might mitigate adverse reactions and prolong the medication period [16]. Our patient did not experience any severe adverse events during 10 cycles of the 2-week regimen of S-1.

In conclusion, we experienced a case of para-aortic and lateral pelvic lymph node recurrence of rectal cancer that showed CR to salvage S-1 monotherapy after the failure of a FOLFOX-4 regimen. S-1 is likely to be an effective, welltolerated and convenient therapeutic option for patients with advanced colorectal cancer.

\section{Disclosure Statement}

The authors have no conflicts of interest to declare.

\section{References}

1. de Gramont A, Figer A, Seymour M, Homerin M,
Hmissi A, Cassidy J, Boni C, et al. Leucovorin and fluorouracil with or without oxaliplatin as first-line treatment in advanced colorectal cancer. J Clin Oncol. 2000;18(16):2938-2947.

2. Cassidy J, Clarke S, Diaz-Rubio E, Scheithauer W, Figer A, Wong R, Koski S, et al. Randomized phase III study of capecitabine plus oxaliplatin compared with fluorouracil/folinic acid plus oxaliplatin as first-line therapy for metastatic colorectal cancer. J Clin Oncol. 2008;26(12):2006-2012.

3. Shirasaka T. Development history and concept of an oral anticancer agent S-1 (TS-1): its clinical usefulness and future vistas. Jpn J Clin Oncol. 2009;39(1):2-15.

4. Ohtsu A, Baba H, Sakata Y, Mitachi Y, Horikoshi N, Sugimachi K, Taguchi T. Phase II study of S-1, a novel oral fluorophyrimidine derivative, in patients with metastatic colorectal carcinoma. S-1 Cooperative Colorectal Carcinoma Study Group. Br J Cancer. 2000;83(2):141-145.

5. Shirao K, Ohtsu A, Takada H, Mitachi Y, Hirakawa K, Horikoshi N, Okamura T, et al. Phase II study of oral S-1 for treatment of metastatic colorectal carcinoma. Cancer. 2004;100(11):2355-2361.

6. Van den Brande J, Schoffski P, Schellens JH, Roth AD, Duffaud F, Weigang-Kohler K, Reinke F, et al. EORTC Early Clinical Studies Group early phase II trial of S-1 in patients with advanced or metastatic colorectal cancer. Br J Cancer. 2003;88(5):648-653.

7. Jeung HC, Rha SY, Cho BC, Yoo NC, Roh JK, Roh WJ, Chung HC, et al. A phase II trial of S-1 monotherapy in metastatic colorectal cancer after failure of irinotecan- and oxaliplatin-containing regimens. Br J Cancer. 2006;95(12):1637-1641.

8. Lee DJ, Lee J, Lee HY, Lim T, Lee SJ, Yi SY, Park SH, et al. Salvage S-1 monotherapy in metastatic colorectal cancer patients who failed irinotecan-based or oxaliplatin-based chemotherapy. Med Oncol. 2011;28(Suppl 1):S291-294.

9. Hoff PM, Ansari R, Batist G, Cox J, Kocha W, Kuperminc M, Maroun J, et al. Comparison of oral capecitabine versus intravenous fluorouracil plus leucovorin as firstline treatment in 605 patients with metastatic colorectal cancer: results of a randomized phase III study. J Clin Oncol. 2001;19(8):2282-2292.

10. Van Cutsem E, Twelves C, Cassidy J, Allman D, Bajetta E, Boyer M, Bugat R, et al. Oral capecitabine compared with intravenous fluorouracil plus leucovorin in patients with metastatic colorectal cancer: results of a large phase III study. J Clin Oncol. 2001;19(21):4097-4106.

11. Douillard JY, Hoff PM, Skillings JR, Eisenberg P, Davidson N, Harper P, Vincent MD, et al. Multicenter phase III study of uracil/tegafur and oral leucovorin versus fluorouracil and leucovorin in patients with previously untreated metastatic colorectal cancer. J Clin Oncol. 2002;20(17):3605-3616. 
12. Shibahara K, Yamamoto M, Kakeji Y, Sakata H, Maehara Y. Retrospective study of S-1 versus tegafur/uracil and oral leucovorin in patients with metastatic colorectal cancer. Anticancer Res. 2008;28(3B):1779-1783.

13. Yamada Y, Tahara M, Miya T, Satoh T, Shirao K, Shimada Y, Ohtsu A, et al. Phase I/II study of oxaliplatin with oral S-1 as first-line therapy for patients with metastatic colorectal cancer. Br J Cancer. 2008;98(6):1034-1038.

14. Zang DY, Lee BH, Park HC, Song HH, Kim HJ, Jung JY, Kim JH, et al. Phase II study with oxaliplatin and S-1 for patients with metastatic colorectal cancer. Ann
Oncol. 2009;20(5):892-896.

15. Muro K, Boku N, Shimada Y, Tsuji A, Sameshima S, Baba H, Satoh T, et al. Irinotecan plus S-1 (IRIS) versus fluorouracil and folinic acid plus irinotecan (FOLFIRI) as second-line chemotherapy for metastatic colorectal cancer: a randomised phase $2 / 3$ non-inferiority study (FIRIS study). Lancet Oncol. 2010;11(9):853-860.

16. Miyazawa T, Ebe K, Koide N, Fujita N. Complete response of liver metastasis of gastric cancer treated by s-1 chemoradiotherapy: a case report. Case Rep Oncol Med. 2012;2012:368428. 